Recherches en didactique des langues et des cultures

Les cahiers de l'Acedle

17-2 | 2020

Recherches collaboratives en didactique des langues

\title{
L'altérité dans la voilure : une lesson study pour un sillage collaboratif en didactiques des langues
}

Carole-Anne Deschoux et Claire Taisson

\section{OpenEdition}

Journals

Édition électronique

URL : https://journals.openedition.org/rdlc/7422

DOI : $10.4000 /$ rdlc.7422

ISSN : $1958-5772$

Éditeur

ACEDLE

Référence électronique

Carole-Anne Deschoux et Claire Taisson, «L'altérité dans la voilure : une lesson study pour un sillage collaboratif en didactiques des langues », Recherches en didactique des langues et des cultures [En ligne], 17-2 | 2020, mis en ligne le 27 avril 2020, consulté le 17 octobre 2022. URL : http:// journals.openedition.org/rdlc/7422 ; DOI : https://doi.org/10.4000/rdlc.7422

Ce document a été généré automatiquement le 17 octobre 2022.

\section{c) (ㄱ)}

Creative Commons - Attribution - Pas d'Utilisation Commerciale - Pas de Modification 4.0 International - CC BY-NC-ND 4.0

https://creativecommons.org/licenses/by-nc-nd/4.0/ 


\title{
L'altérité dans la voilure : une lesson study pour un sillage collaboratif en didactiques des langues
}

\author{
Carole-Anne Deschoux et Claire Taisson
}

1 Dans une institution qui forme des enseignants et qui développe de la recherche, comment et sur quels savoirs construire une relation «collaborative» entre chercheurs/formateurs et enseignants? Que faire, que choisir, en fonction de quoi et de qui ? Peut-on concilier les besoins de formation avec ceux de la recherche?

2 C'est à partir de ces tensions que nous avons initié un questionnement au sein d'une recherche collaborative en nous focalisant sur la construction d'un dispositif. Nous présentons ici les choix posés et les analyses effectuées afin de comprendre la relation construite et les apports réciproques des différents acteurs. Pour ce faire, nous nous référons initialement au concept de recherche collaborative de Desgagné et al., pour qui la réflexivité est au cœur du processus de collaboration. Or la réflexivité se décline différemment selon que l'on est praticien ou chercheur. Lorsqu'on parle de questionnement pratique, les premiers sont projetés dans leur quotidien, et les seconds se réfèrent à des aspects formels. Pourtant cette différence initie et alimente la dynamique même de formation :

À l'issue d'une première étape du projet où chercheurs et praticiens négocient un objet de réflexion commun [...] une activité réflexive [...] sera mise en place. Le déroulement de cette activité réflexive [...] considéré du point de vue des enseignants qui vont y effectuer une démarche d'explicitation et d'analyse de leur pratique en vue de l'améliorer, répond à la définition de ce que Richardson (1994) appelle le « questionnement pratique » et à ce que Beillerot (1991) appelle « être en recherche ». Mais cette même activité réflexive, prise du point de vue des chercheurs qui vont faire de ce matériau réflexif un objet d'analyse en vue de produire des connaissances nouvelles dans un domaine lié à la pratique enseignante et plus spécifiquement au savoir des enseignants, répond à la définition de ce que Richardson (1994) appelle la "recherche formelle» et à ce que Beillerot (1991) appelle «faire de la recherche ». Dans la mesure où les chercheurs du projet se retrouvent du même coup à accompagner et à guider le "questionnement 
pratique » des enseignants, on dit qu'ils jumellent à leur rôle de chercheurs, pris au sens formel du terme, un rôle de formateurs qui encadrent, au fond, la démarche de recherche des enseignants, prise au sens informel du terme. (Desgagné et al, 2001,

pp. 38-39)

3 L'ancrage retenu s'inscrit dans celui de l'enseignement du français. Il est appréhendé selon un paradigme historico-culturel post vygotskien où l'altérité est l'élément structurant de la dialectique du développement. Le développement procède ainsi de l'intersubjectif à l'intrasubjectif (Vygotski, [1934]1997) et est de l'ordre processuel. Ce processus est fait de tensions qui renvoient non seulement aux autres mais à soi-même. L'altérité est ainsi constitutive de cette ipséité (Ricoeur, 1990).

Dans cette contribution est présentée une recherche sur un dispositif de formation dans la discipline français, dispensé à des enseignantes volontaires, en Suisse romande. Il est reconstruit rétrospectivement à partir de traces diverses considérées comme des entités sémiotiques (Radford, 2019). Il porte sur l'usage d'albums en littérature de jeunesse à l'école primaire. L'attention est portée sur ce qui a été élaboré ensemble et sur la dynamique construite entre les différents acteurs. Cette dynamique renvoie à l'espace construit entre les partenaires, aux relations, aux savoirs concernés et aux contraintes rencontrées. Tous ces rapports sont liés aux enjeux des situations considérées, aux groupes et également aux personnes impliquées dans la relation.

Nous présentons d'abord le dispositif de formation qui constitue le cadre de la recherche. Puis nous présentons le support retenu, qui est un album de jeunesse, et ses spécificités. Nous explicitons ensuite ce que les enseignantes et les formatriceschercheures ont effectué ensemble, et les choix posés dans l'usage de l'album en classe. Nous reprenons les éléments qui occasionnent des négociations entre les différents partenaires et qui structurent aussi les échanges. Nous revenons à des éléments configurant ad finem la relation entre enseignantes et formatrices-chercheures.

\section{Présentation de la formation}

\section{Le contexte}

Le contexte retenu est romand. Il se situe dans une institution helvétique qui forme des enseignants. La formation qui nous intéresse s'inscrit en didactique du français comme première langue enseignée (Simard et al., 2010). Elle a été initiée à partir d'un questionnement sur l'usage d'album de jeunesse en classe. Support composite dont la complexité est reconnue (Bautier et al., 2012/2014), l'album de jeunesse est présent massivement dans les classes primaires et également dans les plans d'études. Mais force est de constater que les pratiques rencontrées le plus souvent dans les classes avec ce support visent à enseigner le code ou encore se cantonnent à une lecture oralisée (souvent faite par l'enseignant). La compréhension comme objet d'enseignement est encore trop peu enseignée et constitue une véritable difficulté pour les enseignants (Soussi et al., 2008; Goigoux, 2013; Thévenaz-Christen et al., 2014). De plus, en formation, penser, planifier, mettre en tâches l'enseignement en littérature de jeunesse suscitent également des difficultés chez les étudiants (Deschoux et al., 2015). Mais ces difficultés renvoient aussi à la jeunesse de la discipline littéraire à l'école primaire en suisse romande, à l'hétérogénéité des objets à enseigner, aux référents théoriques qui y sont liés et à leur non articulation (Cordonier, 2012), et également à l'histoire de son 
enseignement en regard de pratiques sédimentées et de leur disciplination (Schneuwly et Dolz, 2009 ; Ronveaux et Schneuwly, 2018).

7 Par ailleurs, dans la même institution du canton de Vaud, une équipe de recherche ${ }^{2}$ développe des travaux en vue d'améliorer les pratiques enseignantes et les apprentissages de tous les élèves par la mise en place de formations outillées de la Lesson Study (Lewis and Hurd, 2011). Elle propose aussi des accompagnements collaboratifs lors de la formation initiale (Clerc et Martin, 2011) et continue.

8 La recherche collaborative sur laquelle nous nous arrêtons ici porte sur un accompagnement proposé à des enseignantes de l'école primaire du cycle 1 (élèves de 4 à 7 ans) voulant travailler avec des albums de littérature de jeunesse dans le cadre d'une formation continue outillée d'une Lesson Study.

Une Lesson Study vise à la fois la construction de nouvelles connaissances sur l'enseignement-apprentissage (Takahashi et McDougal, 2016) et le développement professionnel. L'unité de travail est la leçon. La démarche rassemble des formateurs, des chercheurs et des enseignants (Lewis et Hurd, 2011 ; Takahashi et McDougal, 2016). Elle se caractérise par un processus cyclique: le groupe choisit une thématique et définit des objectifs d'apprentissage, puis il élabore et planifie ensemble une leçon. Après l'avoir planifiée, la leçon est donnée dans la classe de l'un des enseignants sous les yeux du reste du groupe qui l'observe. La leçon est analysée collectivement, a posteriori. Toutes les traces récoltées - matériel utilisé, productions des élèves, photographie instantanée des tableaux noirs, vidéo, etc. - peuvent être potentiellement mobilisées lors des analyses par le groupe, lequel reprend le plan de leçon. Cette nouvelle planification peut donner lieu à une nouvelle leçon donnée et observée dans une autre classe, puis à nouveau analysée, etc. Au terme du cycle, le groupe peut (ou non) documenter à nouveau la leçon et diffuser son travail. La structure de base de la Lesson Study accentue donc la dimension négociée et distribuée des choix posés.

\section{Un dispositif de formation au service de l'enseignement}

10 L'expérience de formation continue dont nous parlons ici est suivie par des enseignantes qui, avec des formatrices-chercheures, définissent des activités et des enseignements à mener à partir d'un album de jeunesse. Cette formation comporte cinq séminaires, deux leçons données devant les collègues et les formatrices-chercheures dans la classe d'une enseignante volontaire à chaque fois différente, deux moments d'échanges juste après les leçons données, lesquels permettent de revenir sur les leçons observées et vécues.

La figure ci-dessous illustre l'organisation de cette Lesson Study. 
Figure 1 - Dispositif de formation

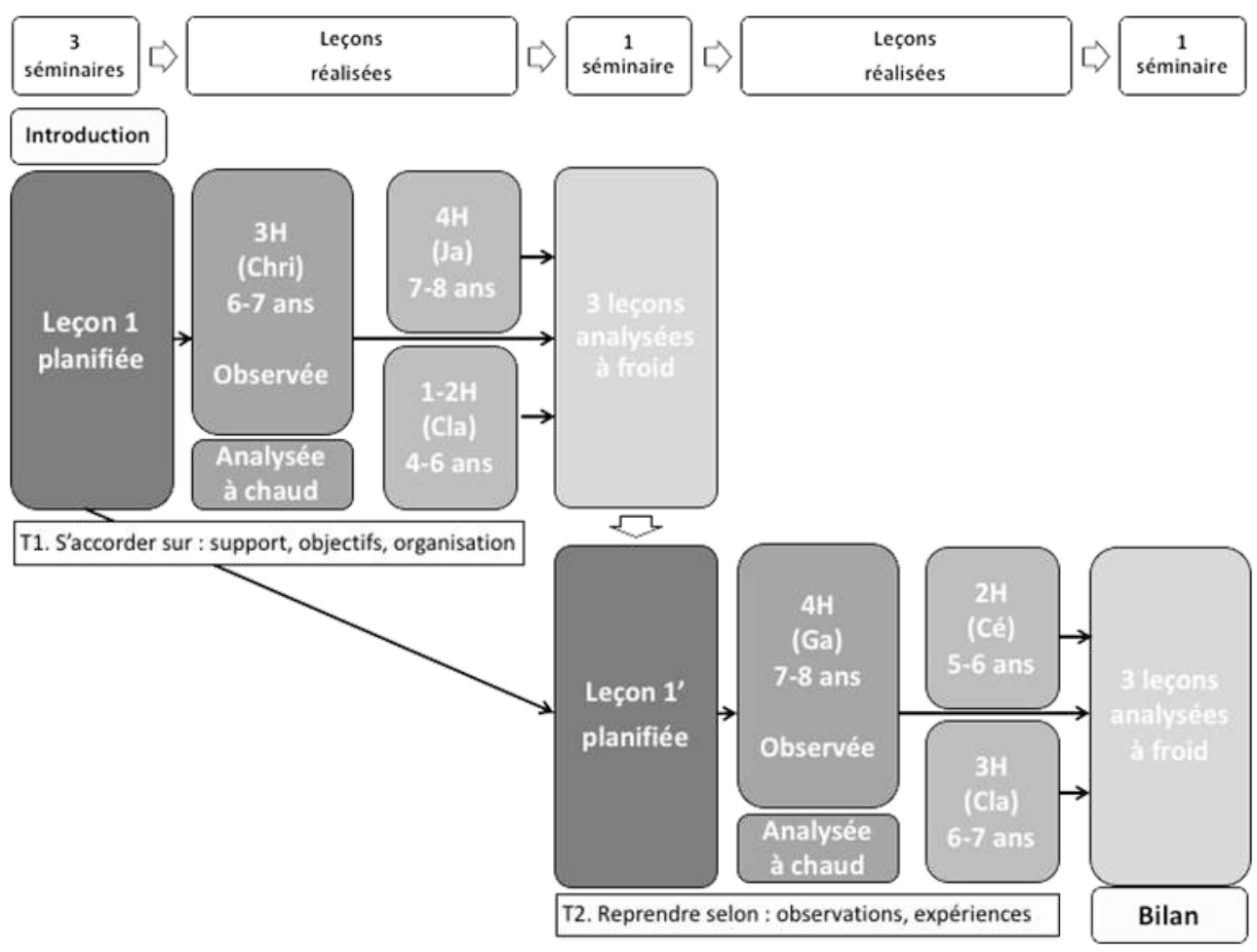

11 Les trois premières séances ${ }^{3}$ (T1) visent à choisir un objet d'enseignement, à définir les modalités de travail et le matériel. Puis une première leçon est réalisée et analysée «à chaud» avec tout le monde (Chri avec des élèves de 6-7ans). De leur côté, deux collègues adaptent et donnent la leçon dans leur classe sans la présence des collègues (Ja avec des élèves de 7-8 ans et Cla avec des élèves de 4-6 ans). La séance suivante reprend l'analyse « à froid » de la leçon et la planification de la nouvelle leçon (T2). La leçon est redonnée par une autre collègue ( $\mathrm{Ga}$ à des élèves de 7-8 ans) et à nouveau analysée « à chaud ». Deux collègues l'adaptent et la réalisent dans leur classe (Cé avec des élèves de 5-6 ans et Cla avec des élèves de 6-7 ans). La formation se termine par une deuxième analyse "à froid » et par un moment de bilan. L'ensemble des sept séances (séminaires et sessions en classe) comporte environ quatorze heures de travail en présentiel.

12 Pour optimiser les échanges et les dimensions expérientielles, les enseignantes assument ainsi différents rôles et responsabilités dans la réalisation et dans l'analyse des leçons. Chaque enseignante peut voir deux leçons, donner une leçon de son côté dans sa classe (sous les yeux ou non des collègues) et alimenter les discussions avec son expérience, ses observations et ses remarques. Les formatrices-chercheures endossent aussi différents rôles; elles gèrent les séances, participent à l'élaboration des leçons, observent les deux leçons données. Elles ont la responsabilité de la formation. Elles gardent en tête les attentes formulées initialement, elles confrontent les points de vue des enseignantes, elles mobilisent leurs observations et amènent des éléments théoriques en fonction de ce qui se présente. 


\section{Un dispositif d'enseignement construit à partir d'un album composite}

Les enseignantes construisent un dispositif d'enseignement ${ }^{4}$ à partir de l'album La soupe au caillou d'Anaïs Vaugelade (2002) - album qu'elles ont choisi ensemble. Pour comprendre les enjeux didactiques de ce choix, il importe de présenter la complexité sémiotique du support. Conte en randonnée ${ }^{5}$, l'ouvrage aborde l'histoire d'un loup qui s'invite chez une poule et lui propose de lui cuire une soupe. Cette visite inquiète les voisins qui heurtent à la porte. Ils rendent visite à la poule les uns après les autres et lui amènent un légume. Ce légume est rajouté à la soupe contribuant au contenu et à la qualité du repas. À la fin, les convives partagent la soupe puis le loup s'en va.

L'album joue sur la tension narrative (Baroni, 2007). L'intertextualité porte sur la réputation du loup, le personnage principal. Cette réputation est explicite dès la première page. Il est écrit que la poule «n'est pas rassurée (...), elle n'a jamais vu de loup en vrai, elle ne le connait que par les histoires ». Sur l'image, le loup a un regard sombre et semble forcer la porte. À plusieurs moments, le lecteur vit cette tension et se demande si le carnivore va manger les convives.

Figure 2 - La soupe au caillou d'Anaïs Vaugelade, 2002 (pp. 5-6)

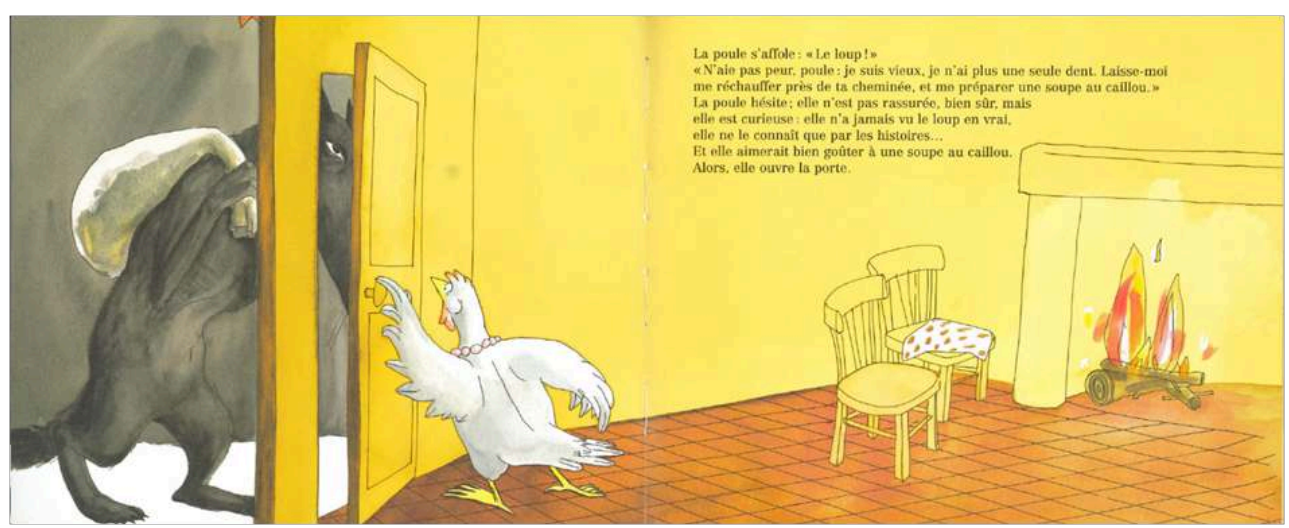

Comme on le voit plus loin (figure 3), le loup a des attitudes ambigües. Sur les images, il est sombre, il regarde en coin, il tient un couteau qu'il dirige vers les hôtes. Dans le texte, il est taiseux.

Figure 3 - La soupe au caillou d'Anaïs Vaugelade, 2002 (pp. 19-20)

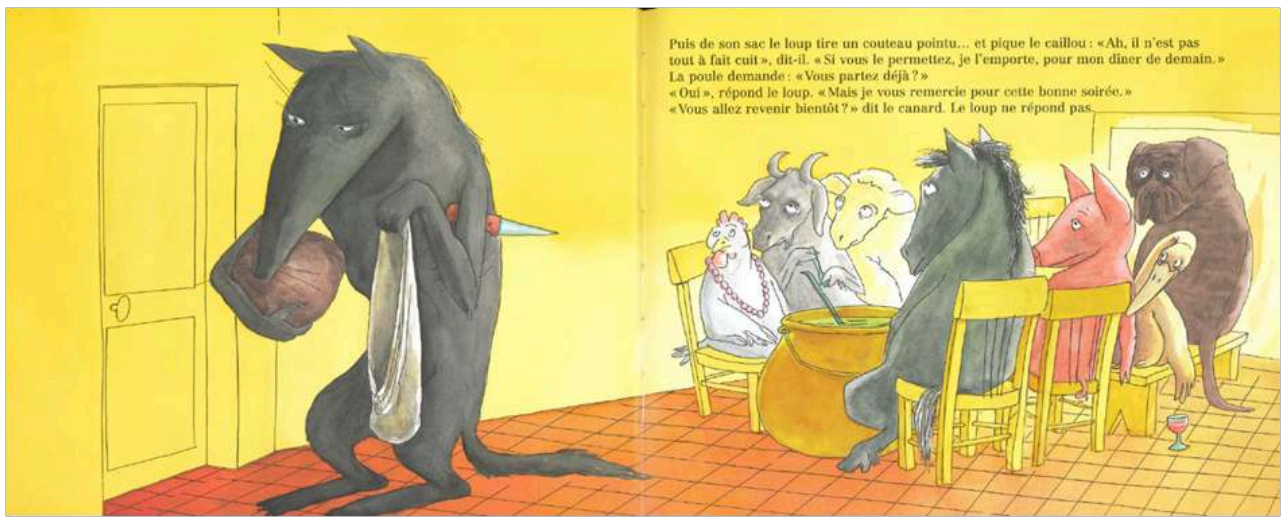


15 Au fil du récit, dans le dialogue de l'image et du texte, l'auteure emmène ainsi le lecteur sur de fausses pistes et l'invite à douter. De plus, au terme de l'histoire, le lecteur reste devant une fin ouverte. Le loup n'a mangé personne. Plusieurs interprétations sont ainsi possibles. Si le lecteur garde la figure du loup, il peut se dire que la ruse a échoué. Mais il peut aussi douter de l'animalité du carnivore en constatant qu'il ne mange jamais et ne manifeste aucune agressivité. Et s'il doute de son animalité, c'est alors une autre figure qui devient saillante: celle du pèlerin. Cette deuxième interprétation renvoie ainsi à une autre version zen de ce conte - qui existe aussi ; par exemple, celle de Jon J. Muth (2011).

Nous avons bien affaire à un support composite dont l'hétérogénéité des traitements et la difficulté interprétative sont manifestes, et dont l'usage ne peut être dissocié de ce que les enseignants comptent en faire avec leurs élèves (Bautier et al, 2010).

\section{Éléments de cadrage épistémologique}

Dans le paradigme interactionniste social que nous adoptons (Bronckart, 1997, 2005), trois dimensions sont mobilisées pour une Lesson Study: 1) l'importance des activités sociales pour le développement personnel des conduites conscientes, 2) le rôle décisif du sémiotique et du langage dans la construction de la pensée consciente, 3) le rôle des médiations formatives dans le processus humain. La trace est ainsi appréhendée comme une entité sémiotique (Radford, 2019) matérielle, pour « ce qu'elle est capable de nous révéler » (p.15). Elle atteste de ce qui s'est passé, s'inscrivant dans un cadre interprétatif qui doit être accompagné d'un appareil méthodologique. Le dispositif d'enseignement construit avec les enseignantes est donc un ensemble structuré d'instruments et de systèmes sémiotiques orientés vers le développement des élèves, et déterminés par une institution scolaire, une discipline et une profession qui ont leur histoire (Schneuwly, 2000).

\section{Éléments méthodologiques}

18 La recherche se base sur la formation continue dispensée à cinq enseignantes volontaires d'une même école primaire du cycle 1 (avec des élèves de 4 à 8 ans). Toutes donnent la leçon à un moment, excepté une enseignante (Cla) qui la donne deux fois avec deux classes différentes (elle travaille dans deux classes). Les planifications, les captations vidéo des leçons, les captations audio d'échanges, les productions écrites des enseignantes (attentes, évaluation), les Power Point, les échanges par email, les productions des élèves constituent le corpus.

19 Le protocole de recherche reconsidère le dispositif de formation à posteriori afin d'identifier et de reconstruire le processus en fonction de ce qui s'est passé. Il est constitué de traces hétérogènes récoltées lors de la formation et des passages dans les classes. De différentes natures, ces traces sont produites en amont ou lors de la formation. Cet appareil méthodologique reconstruit l'entité sémiotique pour pister la dimension explicative. Il suppose de revenir au cadre interprétatif dans lequel on le place (Radford, 2019).

Tous les matériaux font l'objet d'une analyse de contenu. 


\section{Pointages kaléidoscopiques de la relation qui se construit entre les différents partenaires}

\section{Des négociations alternées et des ajouts personnalisés}

En reprenant les choix posés, un subtil jeu de négociations alternées construit l'interdépendance des deux groupes de base (formatrices-chercheures et enseignantes). Il est identifiable au fil des séances et nourrit la dynamique de la relation.

21 Au séminaire 1, les formatrices-chercheures posent le cadre initial (méthodologie, discipline, support, liens avec les prescriptions, nombre de séances). Elles demandent aux enseignantes : 1) de venir avec des albums avec lesquels elles travailleraient en classe, 2) de se préparer à les présenter, 3) de formuler leurs attentes pour la formation. Les enseignantes apportent des albums, chacune verbalise par écrit ses attentes (travailler avec des albums de littérature de jeunesse autrement que par le travail du code, avoir des outils d'analyse de ces albums pour évaluer leur " qualité » et se faire une opinion, pouvoir faire de la place à l'hétérogénéité socioculturelle et plurilingue des élèves, découvrir d'autres albums). Les enseignantes négocient le nombre de séances à la baisse en justifiant que le dispositif est trop impliquant. Le nombre de séances passe ainsi de 13 à 7 séances.

$\mathrm{Au}$ séminaire 2, les albums sont repris. Les formatrices-chercheures abordent les critères d'analyse (dimensions paratextuelles, structuration du texte, situation de communication, contenu de l'histoire, rapport texte-images), l'importance de l'usage du livre en fonction du public à qui on le destine et les apprentissages visés. Les enseignantes relèvent ce qu'elles trouvent intéressant en regard du travail pour leur classe.

23 Entre les séminaires 2 et 3, les formatrices-chercheures demandent aux enseignantes d'effectuer une analyse de l'album. Elles reçoivent des emails des enseignantes dans lesquels elles disent qu'elles ne savent pas la réaliser et qu'elles aimeraient pouvoir la faire ensemble.

24 Au séminaire 3, les formatrices-chercheures reprennent l'analyse de l'album, laquelle est discutée. Les enseignantes construisent une planification (L1). Les choix se font pour la classe dans laquelle la leçon sera réalisée mais doivent être compatibles avec les autres classes. Elle se mettent d'accord sur: 1) la nécessité de travailler la "réputation" du loup pour construire des référents communs entre les élèves et préparer le suspens; en effet, elles relèvent que leurs élèves ne la connaissent pas forcément et que s'ils ne peuvent pas s'y référer, ils ne comprendront pas le danger du potentiel dévoreur; 2) la proposition d'une méthodologie d'investigation pour construire le personnage du loup. Elles constituent ainsi un corpus de cinq livres avec une question qu'elles réservent aux élèves : est-ce que le loup de votre histoire est gentil ou pas? Et pourquoi ? 3) la reprise de cette question avant de débuter la lecture oralisée de l'album par chacune d'elles. Comme le temps prévu en séminaire est trop court, les enseignantes la reprennent de leur côté, en dehors de la formation, et la finalisent sans les formatrices-chercheures.

25 Puis, la première leçon (L1) est donnée dans une classe de 3H (élèves de 6-7 ans). Elle est suivie de son analyse « à chaud ». Pour la première activité, l'enseignante qui donne la leçon fait observer dans les cinq livres, à des groupes de 4-5 élèves, l'attitude, les actes 
des loups, les rapports aux autres personnages. Elle fait constater l'évolution de la menace que peut représenter le carnivore en regard de l'enchainement en succession des pages (Van der Linden, 2013). Elle montre l'aspect « non figé » du loup, en pointant que le loup n'est pas toujours mauvais. La deuxième activité d'oralisation de La soupe au caillou permet bien de réinvestir la réputation du dévoreur potentiel, d'identifier la menace qui plane sur la poule et de vivre la tension narrative liée à ce carnassier. Lors de l'analyse "à chaud", les enseignantes sont satisfaites de ce qu'elles ont vu et vécu. Les enfants ont vécu le suspens. Les formatrices-chercheures relèvent que l'enseignante observée, en effectuant une lecture oralisée où à chaque page elle montrait les images sans questionner le lien entre ce qui était dit et écrit, s'est exclusivement accrochée aux images. Elle a travaillé la compréhension de l'histoire en se basant sur un rapport transparent entre le texte et les images. Les collègues constatent alors qu'elle n'a pas pris en compte les dimensions textuelles et iconographiques comme deux entités distinctes et liées (Van der Linden, 2013) alors qu'elles avaient prévu de le faire dans la planification; l'enseignante observée pensait l'avoir fait dans le déroulement de l'activité. Avec la vidéo, elle a constaté qu'elle a effectivement fait autrement et repris une pratique habituelle avec le postulat que l'image illustrait le texte, et que ce qui figurait dans l'écrit figurait aussi sur les images. Cette façon de faire n'a pas tenu compte de "la "complémentarité" recherchée par certains auteurs-illustrateurs, qui font suivre à l'illustration et au texte des cheminements étanches l'un à l'autre, en évitant toute redondance, [ce qui] engendre certaines difficultés de compréhension " (Canut et Vertalier, 2012, p. 55). L'analyse réalisée ensemble montre que les représentations de ce que l'enseignante pense avoir fait différaient de ce qui avait été effectivement fait et de ce qui avait été décidé collectivement.

En dehors du séminaire, de leur côté, deux enseignantes (Cla et Ja) réalisent l'activité planifiée dans leur classe. Au séminaire 4, l'ensemble du groupe revient sur ce qui s'est passé lors de la leçon observée et sur les leçons données dans les classes des enseignantes (analyse "à froid»). Elles échangent en reprenant les images vidéo, les expériences et les observations. Cla et Ja montrent leur matériel et expliquent les choix posés dans leur classe. Elles ont rajouté des albums, du matériel et ont modifié la consigne. Elles évoquent la nécessité de s'adapter aux élèves et à leur âge. $\mathrm{Cla}^{6}$ avec de très jeunes élèves a d'abord fait observer les caractéristiques du loup en partant d'images diverses du loup figurant sur des cartes pour identifier les similitudes et les différences des représentations. Elle a ainsi pointé avec ses élèves les caractéristiques du loup (grandes dents, griffes, queue). Pour la deuxième leçon, elle a instauré un suspens à partir de premières de couverture d'une collection de cinq albums avec la question « est-ce que le loup est gentil ou méchant?». Elle a relevé que l'on ne pouvait pas se baser sur des caractéristiques et a fait émerger la nécessité de lire le texte pour savoir s'il était méchant ou non. Puis elle a lu l'album de Vaugelade. L'enseignante a donc bien gardé la logique de la progression et le suspens décidés en groupe. 
Figure 4 - Planification de Cla (élèves 4-5 ans) et sa transcription

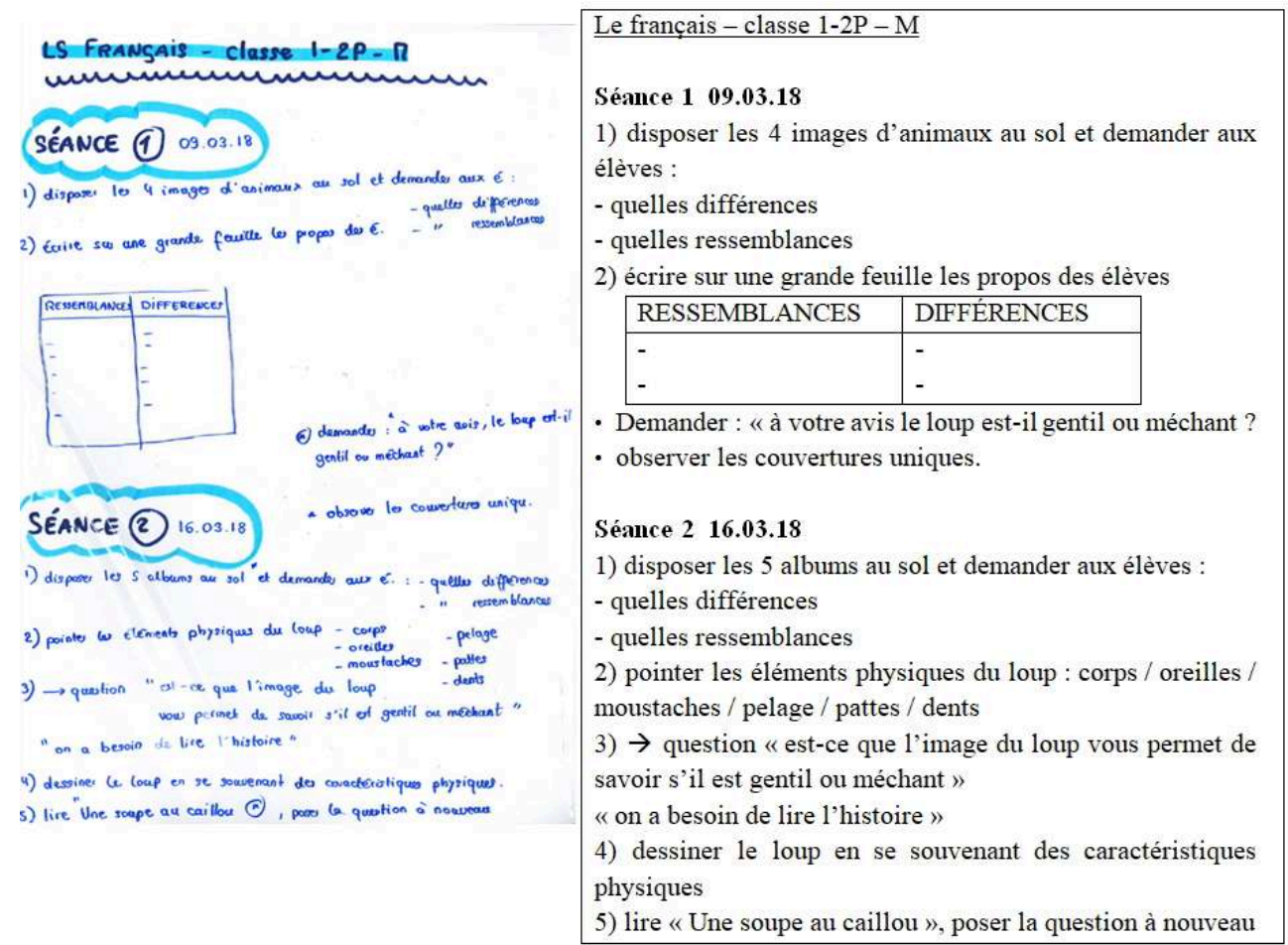

La deuxième enseignante, Ja, a choisi quatre histoires issues d'un même recueil (Aymé, 1973). Elle dit avoir fait la même chose que ses collègues. Elle a proposé une brochure de dix-sept pages téléchargée sur un site (Les coccinelles ${ }^{7}$ ) qui reprenait des exercices visant un foisonnement d'objectifs : travailler les mots outils, identifier et apparier des titres écrits avec différentes typographies, repérer des mots, remplir des textes lacunaires, travailler la fusion syllabique et les phonèmes $/ \mathrm{u} /$ et / $/ \tilde{a} /$, etc. Cette collègue n'est pas rentrée dans ce qui a été proposé par le groupe; elle n'a pas conservé l'importance d'avoir un corpus d'albums, ni de construire l'intrigue. La brochure avec son foisonnement d'exercices traditionnels appuie ce constat. Mais les enseignantes voyant le matériel de Ja demandent à le photocopier. Le groupe, y compris les formatrices-chercheures, ne relève pas le problème. Il reprend la planification 1 , la modifie en fonction des analyses effectuées et de la personne qui a donné la leçon (ici en $3 \mathrm{H}$, élèves de 6 ans).

La leçon (L1') est donnée dans une autre classe par une autre collègue. La collection d'albums, pour la première activité, est composée de quatre albums dont trois nouveaux que l'enseignante en question a choisis. L'analyse "à chaud » permet à tout le monde de constater que l'unité de l'album a été prise en compte et que les aspects textuels et iconotextuels ont été abordés comme deux entités sémiotiques séparées et interdépendantes (van der Linden, 2013). Mais là, un autre problème est alors constaté. Les élèves lors de la leçon font remarquer qu'on ne peut pas dire qu'un loup est méchant s'il doit se nourrir, car un animal doit manger pour vivre. Les formatriceschercheures reprennent ce qu'ils disent et pointent l'évolution et la complexité des personnages dans le récit et l'importance du genre narratif. Elles posent le statut de la fiction. Le personnage du loup dans un récit est anthropomorphisé. Il est doté d'intentionnalité comme les êtres humains. Il peut être méchant. Alors que la fiction implique une rupture par rapport au vrai loup. Dans la réalité, comme animal, il doit 
effectivement se nourrir et ne peut être considéré comme " méchant ». Ensemble, elles constatent que même si les loups sont souvent des dévoreurs, même s'ils ont mauvaise réputation, d'une part ils ne sont pas tous "méchants " dans les textes retenus et, d'autre part, certains loups évoluent au fil du texte en regard de la dynamique du récit. Toutes reprennent le corpus pour revenir au personnage du loup dans le récit de Vaugelade. Des élèves avaient dit qu'il était gentil car il n'avait pas mangé les animaux ou alors parce qu'il avait cuit une soupe. Elles constatent l'impossibilité de savoir si ce loup est gentil ou méchant. Elles se demandent si sa ruse a échoué, car il y avait finalement trop d'invités pour manger la poule, ou s'il n'avait plus faim. Les formatrices-chercheures font émerger que la figure du loup est en tension avec celle du pèlerin qui existe aussi dans d'autres versions de cette même histoire. Elles montrent un album qui est un conte zen appelé la soupe aux cailloux de Jon J. Muth (2011). Ici des moines zen proposent à des villageois de faire une soupe afin de recréer des liens de solidarité entre eux. Le loup n'est donc peut-être pas la seule figure de Vaugelade. Son loup ressemble à un pèlerin qui va de maison en maison pour créer de la solidarité entre les habitants alors qu'il fait froid dehors. Pour le comprendre, il faut comparer les deux figures en effectuant un travail d'observation et de lexique de manière à les qualifier, à les caractériser. Il s'agit aussi de considérer ce qui est dit sur lui et les réactions qu'il provoque auprès des autres personnages. Ce rapprochement entre les deux histoires de soupe aux cailloux permet d'évoquer d'autres histoires de soupe aux cailloux (il en existe plusieurs versions, car il s'agit d'un conte populaire).

Puis, à nouveau, deux enseignantes donnent la leçon dans leur classe. Cla (6-7 ans) et Cé (5-6 ans) choisissent un corpus de cinq albums. Cla garde la même planification avec des élèves élèves d'une année de plus.

Le séminaire 5 est consacré à l'analyse "à froid " du deuxième tour. Le contenu, les dimensions socioculturelles et plurilingues sont repris avec la question des adaptations et des réécritures en regard des différentes versions du conte et des multiples histoires de loup. Il est relevé l'importance de travailler la mise en réseau des différents récits afin de toucher et d'enrôler un public dont les référents socioculturels pourraient potentiellement différer et de montrer les variations. Elles reprennent les attentes, le dispositif et les outils d'analyse construits. Les enseignantes sont toutes ravies de cette formation et relèvent qu'elles ont beaucoup appris. Une dit quand même regretter ne pas avoir eu plus d'échanges autour des albums présentés au début. Ja dit avoir apprécié mais ne rendra jamais son évaluation écrite aux formatrices-chercheures. Ces dernières sont aussi contentes des échanges mais se demandent si les collègues arriveront à reproduire seules ce genre de démarche.

\section{Le dispositif de formation au service des apprentissages des élèves}

31 Le dispositif de formation est en cohérence avec ce qui a été annoncé. Par les choix posés, les initiatives assumées, formatrices-chercheures et enseignantes sont bien orientées vers les dimensions pragmatiques liées au terrain et visent les apprentissages à faire construire par les élèves. Les deux groupes sont engagés dans la relation; tout le monde est présent aux séances, les activités sont réalisées et du matériel est échangé. Les enseignantes prennent du temps en dehors du temps de formation pour négocier et stabiliser la planification, pour chercher des livres. Les formatrices-chercheures reviennent sur les éléments pointés, à l'aide de matériel, tentent de répondre aux attentes et aux besoins des collègues et interviennent sur les contenus. Les deux 
groupes de professionnelles sont solidaires et réunis autour d'un premier but qui est le travail en classe.

Ce même but est assumé en regard de responsabilités distribuées. Ce qui se passe réellement en classe est pris en charge par les enseignantes. À la fois elles doivent tenir compte des choix collectifs tout en les adaptant à la particularité de leur classe.

Les formatrices-chercheures acceptent ces initiatives tout en ayant le souci du maintien des objectifs de formation et de la réalisation des attentes verbalisées des enseignantes. Elles cherchent à encourager l'enthousiasme des enseignantes tout en assumant la reprise de décalage en regard du contenu enseigné. Elles mènent l'analyse de pratique. Cette analyse est aussi outillée par les résultats de recherche et leurs connaissances scientifiques.

La responsabilité est ainsi partagée et distribuée en fonction des compétences, des connaissances professionnelles des deux groupes.

\section{Des groupes labiles pour jouer des frontières et des enjeux}

Même si initialement, il y a un groupe de formatrices-chercheures et des enseignantes en formation, d'autres groupes labiles se forment en fonction des enjeux de la situation, du moment et de l'avancée du processus. Il y a un jeu de frontières entre les personnes qui constituent ces groupes. Trois moments forts ont été repérés.

Le premier moment (séminaires 1, 2,3) comporte un enjeu important qui est celui de donner envie de s'engager tout en identifiant comment. Il vise à définir la place des unes et des autres et les responsabilités à assumer par rapport au travail en classe et à la formation continue.

ầme moment est décliné en deux parties qui est la mise à l'épreuve de ce qui a été construit en commun dans des contextes d'enseignement différents. Il montre que la leçon commune de lecture oralisée de l'album est partout la même, mais que c'est la leçon qui précède qui varie. Cette variation renvoie à la particularité de la classe, du matériel et de l'enseignante. Il permet aussi de constater que ce qui est pensé être effectué n'est pas forcément ce qui est fait et que ce qui est fait peut être lié à quelque chose d'intériorisé (pratique séculaire ou habitus) que l'on n'interroge pas forcément.

Le troisième moment permet une resaisie de ce qui s'est passé et un positionnement par rapport à ce qui a été vécu et appris.

Ces trois moments renvoient à des frontières qui délimitent des sous groupes: les collègues des degrés enseignés (1-2 versus 3-4); celles qui enseignent devant les autres versus celles qui regardent; celles qui donnent la leçon versus celles qui ne l'ont pas essayée ou qui ne l'essayeront jamais. Ces groupes impliquent des jeux de forces, des rôles différents et supposent des échanges physiques et des verbalisations qui nourrissent les dimensions personnelles et collectives. Ces tensions contribuent à la dynamique du processus et ont des répercussions potentielles sur chacune des personnes. 


\section{Un processus nourri par des jeux et des enjeux entre formatrices- chercheures et enseignantes}

La dimension fondamentalement sociale des conduites conscientes à l'œuvre dans la visée d'assurer et d'améliorer ce qui est appris aux élèves est le liant de la relation. C'est à cette condition que les enseignantes acceptent de se trouver sous tension et qu'elles s'investissent, assument leur rôle dans la Lesson Study et se forment potentiellement. C'est aussi à l'aune de ces échanges que les formatrices-chercheures comprennent la complexité du métier d'enseignant en classe, et qu'elles se transforment aussi. Le processus et la transformation sont identifiables par le dispositif de formation qui se co-construit et se négocie entre les participantes. Certains choix peuvent être considérés «hybrides» du côté de la recherche. Ils sont totalement assumés. Le dispositif de formation fait ainsi aussi bouger les frontières épistémologiques et invite à les dépasser. Le processus engagé se décline donc à différents niveaux et à différents moments de la formation-recherche. Il invite à revenir au concept d'altérité qui se saisit à différents niveaux et qui constitue son moteur fondamental.

Niveau de la Lesson Study:

41 La Lesson Study construit le rapport aux différentes altérités en présence dans la formation et dans la recherche mais aussi par la formation et par la recherche. Par le caractère cyclique (de reprise des planifications, des leçons, des échanges), par la dimension partagée (de la construction des planifications, de l'essai des leçons) et distribuée (dans la répartition de ce qu'il y a à faire), toutes les participantes se sentent concernées et responsables. Elles se mettent en danger parfois dans ce qu'elles montrent « d'imparfait» ou dans ce qu'elles voient comme différent chez les autres. Par ces échanges, mais aussi par les aspects techniques (vidéos, documents écrits), le processus est couteux. Se voir en vidéo n'est pas forcément facile; reprendre ce qui a été fait et le repenser dans un collectif est éprouvant - même si la bienveillance est présente.

Niveau des classes:

42 Pour les classes, une rupture est constatée dans le travail effectué par les enseignantes entre les deux premières années $(1 \mathrm{H}-2 \mathrm{H}$, élèves de $4-6$ ans) et les deux suivantes (3H-4H, élèves de 6-8 ans), mais aussi entre celles qui sont observées et les autres. Même si les enseignantes construisent une même planification, gardent les mêmes objectifs, elles adaptent le choix des livres pour le corpus; elles modifient le matériel. Le dispositif de formation permet de montrer l'adaptation aux élèves; il permet d'articuler les niveaux individuel et collectif.

Niveau de la formation:

43 Les altérités évoquées en fonction des groupes labiles sont un vecteur dynamique de la relation entre les différentes participantes, marquée par des enjeux de groupes, des enjeux personnels (formation - recherche - enseignement - apprentissage) en tension avec des enjeux communs (le travail en classe, l'engagement des unes par rapport aux autres).

44 Ce processus est construit par de la sémiotisation, car il est investi, transformé par les différents acteurs. Il renvoie aux traces appréhendées comme des entités sémiotiques (Radford, 2019) matérielles, par rapport «à ce qu'elles nous donnent à voir». Le 
principe dynamique est identifiable par les traces matérielles laissées dans la succession des étapes du dispositif de formation. Il est nourri par le jeu de tensions entre les différents groupes et rôles assumés par les différents partenaires.

\section{Conclusion}

Dans cette recherche, nous nous sommes intéressées au processus construit par et à travers un dispositif de recherche-formation collaborative outillé d'une Lesson Study, ainsi qu'à la dynamique construite entre les participantes.

Nous avons montré le caractère distribué et situé de ce qui s'est développé et nous avons relevé l'importance des enjeux et des rôles liés à chaque étape du dispositif et aux participantes.

47 Le dispositif de formation a bien le statut d'instrument (Schneuwly, 2000) qui permet, initie, contraint le travail entre des professionnels. Il invite les enseignantes à transformer pour leurs classes ce qui est négocié collectivement tout en gardant le cap de ce qui a été décidé. Cette adaptation rend compte de la sémiotisation qu'elles construisent dans et par cette adaptation, mais aussi des contraintes liées à leur environnement. Elle permet ainsi, par la matérialité, d'attester de ce qui s'est fait, de le reprendre et de le reconfigurer tout en remobilisant les représentations des unes et des autres. L'action observée est ainsi tracée et discutée.

Dans notre dispositif de formation, nous constatons que le rapport à l'autre est central, multiforme et se manifeste à différents niveaux. Ce rapport à l'autre renvoie à une multitude d'altérités qui renvoient à des rôles définis, mais aussi à des groupes labiles qui sont des moteurs de développement.

Ainsi, ces altérités permettent d'accompagner le processus et engendrent du développement. Ces altérités permettent d'entretenir un principe dynamique impliquant un jeu de frontières entre différents groupes labiles. Ces altérités jouent des tensions et des enjeux du moment.

La collaboration entre professionnelles permet ainsi de s'inscrire dans un réseau plus large qui contribue à alimenter une dynamique sociale de collègues intéressés et impliqués par le travail scolaire, lequel va bien au-delà du scolaire... Les besoins et les enjeux de formation et de recherche peuvent être conciliés, même s'ils sont aussi en tension. La poursuite du processus suppose un délicat assemblage où chacune a sa place sur le bateau...

\section{BIBLIOGRAPHIE}

Baroni, R. (2007). La tension narrative. Suspense, curiosité, surprise. Paris : Seuil. 
Bautier, E. Crinon, J. Delarue-.Breton, C et Marin, B. (2012). Les textes composites : des exigences de travail peu enseignés ? Repères, 45, mis en ligne le 15 juin 2014. http://reperes.reveues.org/ 136, consulté le 17 décembre 2019.

Beillerot, J. (1991). La « recherche », Essai d'analyse. Recherche et formation, 9, p. 17-31.

Bronckart, J.-P. (1997). Activité langagière, textes et discours. Pour un interactionnisme socio-discursif. Paris : Delachaux \& Niestlé.

Bronckart, J.-P. (2005). Les différentes facettes de l'interactionnisme socio-discursif, Calidiscópio, 3, p. 149-159. https://archive-ouverte.unige.ch/unige :37301, consulté le 17 décembre 2019.

Canut, E. et Vertalier, M. (2012). Lire des albums : quelle compréhension et quelle appropriation par les élèves de maternelle? Le français aujourd'hui, 179, p. 51-66. Paris : Armand Colin.

Clerc, A. et Martin, D. (2011). L'étude collective d'une leçon, une démarche de formation pour développer et évaluer la construction des compétences professionnelles des futurs enseignants, Revue en ligne de pédagogie de l'enseignement supérieur Ripes, mis en ligne le 16 janvier 2012. Disponible à : https://journals.openedition.org/ripes/514, consulté le 11 février 2014.

Cordonier, N. (2012). Un curriculum postmoderne. Dans J.-L. Dumortier, J. Van Beveren et D. Vry daghs (ed.), Curriculum et progression en français. Actes $d u$ 11 ${ }^{e}$ colloque de l'AIRDF (Liège, 26-28 aout 2010) (pp. 22-34). Namur : PUN.

Deschoux, C.-A., Florey, S. et Ronveaux, C. (2015). Sous les pavés de la tâche, la plage des savoirs scolaires et la littérature. Revue des hautes écoles pédagogiques de la Suisse Romande et du Tessin, 19, 127-136.

Desgagné, S., Bednarz, N., Couture, C., Poirier, L. et Lebuis, P. (2001). L'approche collaborative de recherche en éducation : un rapport nouveau à établir entre recherche et formation, Revue des sciences de l'éducation, XXVII/1, p. 33-64.

Goigoux, R. (2013). Lire et écrire, rapport de recherche, étude de l'influence des pratiques de l'enseignement de la lecture et de l'écriture sur la qualité des premiers apprentissages. Nyon : IFE.

Laboratoire Lausannois Lesson Study, HEP Vaud : https://www.hepl.ch/cms/accueil/formation/ unites-enseignement-et-recherche/enseignement-apprentissage-eval/laboratoire-lausannoislesson-st.html, consulté le 30 janvier 2020.

Lewis, C. et Hurd, J. (2011). Lesson study step by step : How teacher learning communities improve instruction. London : Heinemann Dedicated to Teachers.

Conférence Intercantonale de l'Instruction Publique de la Suisse romande et du Tessin (CIIP) (2010-2016) Plan d'études romand. Neuchâtel : Secrétariat général de la CIIP. Disponible à : https:// www.plandetudes.ch, consulté le 29 janvier 2020.

Radford. L. (2019). Trace, ontologie, politique et apprentissage. Dans S. Boéchat-Heer et C. Ronveaux, La trace dans les recherches sur la formation et l'enseignement, Hors-série, nº3, (pp. 15-31). Fribourg : Cahr. Version informatique : http://revuedeshep.ch/pdf/HS3/HS3-02-Radford.pdf, consulté le 17 décembre 2019.

Richardson, V. (1994). Conducting research on practice. Educational Researcher, 23(5), p. 5-10.

Ricoeur, P. (1990). Soi-même comme un autre. Paris : Seuil.

Ronveaux, C. et Schneuwly, B. (2018). Lire des textes réputés littéraires : disciplination et sédimentation. Enquête au fil des degrés scolaires en Suisse romande (Théocrit). Berne : Peter Lang.

Schneuwly, B. (2000). Les outils de l'enseignant - Un essai didactique. Repères, recherches en didactique du français langue maternelle, 22. Les outils d'enseignement du français, sous la direction 
de Sylvie Plane et Bernard Schneuwly. pp. 19-38. Disponible à : www.persee.fr/doc/ reper_1157-1330_2000_num_22_1_2341, consulté le 30 janvier 2020.

Schneuwly, B. et Dolz, J. (2009). Les objets enseignés en classe de français. Rennes : PUR.

Simard, C., Dufays, J.-L. et Garcia-Debanc, C. (2010). Didactique du français langue première. Bruxelles : de Boeck.

Soussi, A., Petrucci, F., Ducrey, F. et Nidegger, C. (avec la participation de Ferrez, E. et Schwarz, F.) (2008). Les pratiques déclarées d'enseignement de la lecture et performances des élèves dans le canton de Genève. République et canton de Genève : Service de la recherche en éducation.

Takahashi, A., McDougal, T. (2016). Collaborative lesson research : maximizing the impact of lesson study. ZDM Mathematics Education, p. 1-14.

Thévenaz-Christen, T., Aeby Daghé, S., Jacquin, M., Leopoldoff Martin, I. Ronveaux, Chr., Sales Cordeiro, Gl., Soussi, A., Wirthner, M. (2014). La lecture enseignée au fil de l'école obligatoire. L'exemple genevois. Namur : PUN.

Van der Linden, S. (2013). Album(s). Paris : de facto.

Vygotski, L.S. (1934/1997). Pensée et langage. Paris : La Dispute.

\section{Albums}

Aymé. M. (1973). Les contes du chat perché. Paris : Gallimard.

Muth, Jon J. (2011). La soupe aux cailloux. Paris : Circonflex.

Vaugelade, A. (2002). La soupe au caillou. Paris : École des loisirs.

\section{NOTES}

1. La formation a rassemblé uniquement des femmes. C'est donc le féminin qui est adopté.

2. Laboratoire 3LS : Laboratoire Lausannois Lesson Study. https://www.hepl.ch/cms/accueil/ recherche/laboratoires-hep-vaud/3ls.html

3. Le terme séance désigne ici une session de formation de deux heures. Il désigne soit un séminaire, soit une session dans une classe pour laquelle toute l'équipe est présente.

4. Pour préciser, le dispositif d'enseignement est destiné à être implanté dans la classe, il est élaboré en vue des apprentissages des élèves; alors que le dispositif de formation fédère les enseignantes en formation et les chercheures.

5. Un conte en randonnée est construit sur une structure répétitive, cumulative et simple.

6. Sur la figure 4 , il apparait que le terme séance désigne ce que nous avons appelé leçon.

7. Site: http://www.les-coccinelles.fr, et les exercices liés à l'album: http://www.lescoccinelles.fr/lienpage1/albums/unesoupeaucaillou/litteraturejeunesseunesoupeaucaillou.html, consultés le 23 avril 2020. 


\section{RÉSUMÉS}

Cette contribution s'inscrit en didactique du français langue première. Elle se focalise sur l'analyse d'un dispositif de formation et cherche à montrer le principe dynamique construit par la collaboration d'enseignantes et de chercheures ${ }^{1}$ dans le cadre d'une formation continue dispensée dans un institut de formation. Elle rend compte d'une recherche collaborative (Desgagné et al., 2001) outillée d'une Lesson Study (Lewis et Hurd, 2011). Dans cette analyse à caractère exploratoire, le dispositif de formation et sa transformation permettent à la fois de reconstruire le processus de collaboration entre les chercheures et les enseignantes, et de repartir de cette collaboration pour revenir au dispositif en question. Cette recherche montre comment, en fonction des enjeux épistémologiques, des savoirs et des expériences communes et personnelles, ces professionnelles de la recherche et du terrain construisent d'une manière distribuée et partagée cet artéfact, et comment ce dernier permet des déclinaisons variables qui peuvent être réinvesties dans les différentes classes des enseignantes concernées.

This contribution is part of the teaching of French. It focuses on the analysis of a system and seeks to show the dynamic principle built by the collaboration of teachers and researchers in the context of continuing training provided in a training institute. It reports on collaborative research (Desgagné et al., 2001) using a Lesson Study device (Lewis and Hurd, 2011). In this exploratory analysis, the artefact and its transformation make it possible to reconstruct the process of collaboration between researchers and teachers and the collaboration in turn makes it possible to return to the artefact in question. This research shows how, based on epistemological issues, common and personal knowledge and experiences, these research and field professionals build this artifact together and how it allows for variable variations that make it possible to reinvest in the various classes of the concerned teachers.

\section{INDEX}

Mots-clés : altérité, Lesson Study, positionnement historico-culturel, didactique des langues

Keywords : otherness, Lesson Study, historical-cultural positioning, language didactics

\section{AUTEURS}

\section{CAROLE-ANNE DESCHOUX}

Haute école pédagogique (HEP) du canton de Vaud, Lausanne

Carole-Anne Deschoux a une formation et une expérience d'enseignante à l'école primaire et en enseignement spécialisé. Ses recherches s'inscrivent dans un ancrage historico-culturel post vygotskien. Son appréhension des questions didactiques, sur l'apprentissage du français notamment, est marquée par ce parcours avec une attention particulière aux dimensions plurilingues.

carole-anne.deschoux[at]hepl.ch

\section{CLAIRE TAISSON}

Haute école pédagogique (HEP) du canton de Vaud, Lausanne

Longtemps enseignante et directrice d'établissements primaires français, les recherches de Claire Taisson s'inscrivent dans le paradigme vygotskien. Elle étudie comment les élèves des premiers 
degrés construisent leurs connaissances au moyen de la matérialité vue comme ressource pour l'enseignement-apprentissage et comme indice de leur développement psychologique.

claire.taisson[at]hepl.ch 